\title{
Addendum to: Dynamical system analysis of a three fluid cosmological model: an invariant manifold approach
}

\author{
Subhajyoti Pal ${ }^{1, \mathrm{a}} \mathbb{C}$, Subenoy Chakraborty ${ }^{2, \mathrm{~b}}$ \\ ${ }^{1}$ Department of Mathematics, Sister Nibedita Govt General Degree College For Girls, Kolkata, West Bengal 700027, India \\ ${ }^{2}$ Department of Mathematics, Jadavpur University, Kolkata, West Bengal 700032, India
}

Received: 1 April 2020 / Accepted: 2 April 2020 / Published online: 21 May 2020

(C) The Author(s) 2020

\section{Addendum to: Eur. Phys. J. C (2019) 79:362 https://doi.org/10.1140/epjc/s10052-019-6875-4}

We the authors want to further mention that the three fluid cosmological model has been considered before in some earlier works. Especially in [1], to determine the viable nature of the scalar field potential, the authors applied an extra Lie symmetry to the dynamical system describing the model to conclude that the scalar field potential should be exponential. This is in accordance with our assumption. In [2], the author applied the Liyapunov methods to construct the Liyapunov functions in some of the non-hyperbolic cases to demonstrate the stability of the non-hyperbolic critical points. In contrast, in our paper we used the Center Manifold Theory to construct the center manifolds and the reduced systems to all the non-hyperbolic cases to demonstrate the stability. Although both the papers deal with essentially the same model, the methods incorporated are entirely different in their own merit.

Data Availability Statement This manuscript has no associated data or the data will not be deposited. [Authors' comment: This is an addendum of our original article. This addendum does not contain any data as it doesn't need to but the original article contains all the data relevant to our research.]

Open Access This article is licensed under a Creative Commons Attribution 4.0 International License, which permits use, sharing, adaptation, distribution and reproduction in any medium or format, as long as you

The original article can be found online at https://doi.org/10.1140/ epjc/s10052-019-6875-4.

${ }^{a}$ e-mail: palsubhajyoti@gmail.com (corresponding author)

be-mail: schakraborty.math@gmail.com give appropriate credit to the original author(s) and the source, provide a link to the Creative Commons licence, and indicate if changes were made. The images or other third party material in this article are included in the article's Creative Commons licence, unless indicated otherwise in a credit line to the material. If material is not included in the article's Creative Commons licence and your intended use is not permitted by statutory regulation or exceeds the permitted use, you will need to obtain permission directly from the copyright holder. To view a copy of this licence, visit http://creativecomm ons.org/licenses/by/4.0/.

Funded by $\mathrm{SCOAP}^{3}$.

\section{References}

1. M. Tsamparlis, A. Paliathanasis, Three fluid cosmological model using Lie and Noether symmetries. Class. Quant. Grav. 29(1) (2011). arXiv:1111.5567 [astro-ph]

2. M. Azreg-Aïnou, Phase-space analysis of the cosmological 3-fluid problem: families of attractors and repellers. Class. Quant. Grav. 30, 205001 (2013). arXiv:1304.7470v3 [astro-ph] 